\title{
Scenario of Extended Spectrum Beta Lactamase (ESBL) producing Escherichia coli in a rural medical college of Himachal Pradesh
}

\author{
Dr Anuradha Sood ${ }^{1}$, Dr Lata R Chandel ${ }^{2}$, Dr Smriti Chauhan ${ }^{3}$, \\ Dr Kamlesh Thakur ${ }^{4}$, Dr Subhash Jaryal ${ }^{5}$ \\ ${ }^{1}$ Assistant Professor, Department of Microbiology, Dr Rajendra Prasad Medical College, Kangra at Tanda, (H.P). \\ ${ }^{2}$ Senior Resident, Department of Microbiology, Dr Rajendra Prasad Medical College, Kangra at Tanda, (H.P). \\ ${ }^{3}$ Senior Resident, Department of Microbiology, Dr Rajendra Prasad Medical College, Kangra at Tanda, (H. P). \\ ${ }^{4}$ Professor and Head, Department of Microbiology, Dr Rajendra Prasad Medical College, Kangra at Tanda,(H.P). \\ ${ }^{5 .}$ Associate Professor, Department of Microbiology, Dr Rajendra Prasad Medical College, Kangra at Tanda, (H.P).
}

\begin{abstract}
Extended Spectrum Beta Lactamases (ESBLs) are beta-lactamases that hydrolyze extended-spectrum cephalosporins. They confer resistance to all $\beta$-lactam antibiotics with the exception of carbapenems. ESBL-producing organisms may appear susceptible to some extended-spectrum cephalosporins in routine antibiotic susceptibility testing. The detection of ESBL's in 175 clinical isolates of Escherichia coli was done by a screening test, followed by comparison between two confirmatory tests i.e. double disc synergy test (DDST) and the Disc on disc (DOD) test. ESBL production was detected by DDST in 45\% and by DOD in $23 \%$ of cases. The antibiotic susceptibility pattern by Kirby Bauer technique showed that $54 \%$ isolates were resistant to all the six first line antibiotics. Maximum drug resistance with ampicillin and no resistance with meropenem was seen. The results show that there is a high frequency of ESBL producing E.coli in our hospital . We recommend that an appropriate antibiotic policy should be framed in every hospital to check for indiscriminate use of drugs and that ESBL detection by DDST should be mandatory for every laboratory as it a easy and convenient way to detect drug resistance.
\end{abstract}

Keywords: Extended Spectrum Beta Lactamases, double disc synergy test, Disc on disc, Escherichia coli.

\section{INTRODUCTION}

The occurrence of Extended Spectrum Beta Lactamases (ESBL) in members of family Enterobacteriaceae is increasingly being reported worldwide ${ }^{[1,2]}$. Generally, they confer resistance to most beta lactams like penicillins, cephalosporins and monobactams, with the exception of cephamycins (cefoxitin and cefotetan) and carbapenems ${ }^{[3]}$. The ESBLs are frequently plasmid encoded. Plasmids responsible for ESBL production frequently carry genes encoding resistance to other drug classes (for example, aminoglycosides). Therefore, antibiotic options in the treatment of ESBL-producing organisms are extremely limited. Detection of ESBL producing organisms is a challenge for laboratories since routine methods of antibiotic susceptibility testing are not sensitive enough to detect them. ESBLs can be detected by disc diffusion methods, three dimensional agar tests, rapid automated systems, E-test and Polymerase chain reaction ${ }^{[4]}$. The aim of this study was to find out the frequency of ESBL production in E. coli, their resistance pattern in vitro and to compare two disc diffusion based tests for the detection of ESBL in our part of the state as no such study has been done here previously.

\section{MATERIAL AND METHODS}

A total of 175 isolates of $E$. coli from various clinical specimens, were obtained and identified by standard microbiological techniques ${ }^{[5]}$. Antibiotic susceptibility testing (AST) was performed on Mueller Hinton agar (MHA) by Kirby Bauer technique ${ }^{[6]}$. Screening for ESBL production was done as per criteria recommended by Clinical Laboratory Standards Institute (CLSI) by using Ceftazidime $(30 \mu \mathrm{g})$ disc in each case ${ }^{[7]}$. An inhibition zone of $\leq 22 \mathrm{~mm}$ for ceftazidime was considered as an indication of ESBL production. 100 isolates of E.coli found to be positive in screening test were then subjected to confirmatory tests for ESBL production which included the Double disc synergy test (DDST) and Disc on disc (DOD) method. DDST was carried out by swabbing approximately $5 \times 10^{5} \mathrm{CFU} / \mathrm{ml}$ of each isolate on MHA. Ceftazidime (30 $\mu \mathrm{g}$ ) disc and a co-amoxiclav ( $20 \mu \mathrm{g}$ of amoxicillin and $10 \mu \mathrm{g}$ of clavulanic acid) disc were placed apart on MHA surface in such a manner that the distance between the two discs was approximately twice the zone of inhibition (ZOI) produced around the ceftazidime disc in screening method ${ }^{[8]}$. Co-amoxiclav discs were used as a source of beta lactamase inhibitor clavulanic acid. The agar plates were incubated at $37^{\circ} \mathrm{C}$ for 18 hours. The organism was considered as ESBL producer if the zone of inhibition around the Ceftazidime disc showed an extension towards the co-amoxiclav disc. (Fig. 1) 


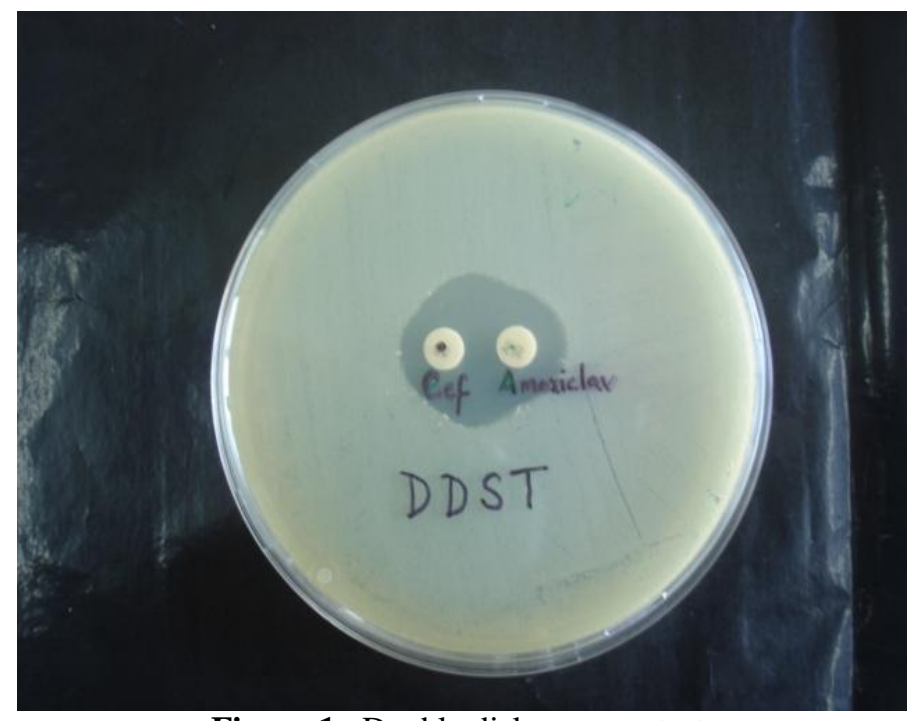

Figure 1 -Double disk synergy test.

In the DOD method a lawn culture of approximately $5 \times 10^{5} \mathrm{CFU} / \mathrm{ml}$ was made on the surface of MHA. Ceftazidime disc overlaid carefully with co-amoxiclav disc was placed $25 \mathrm{~mm}$ away from another Ceftazidime disc ${ }^{[9]}$. The agar plates were then incubated at $37^{\circ} \mathrm{C}$ for 18 hours. A test was considered positive if the difference in diameters of ZOI of Ceftazidime disc in combination with co-amoxiclav disc and Ceftazidime disc alone was $\geq 5 \mathrm{~mm}$ (Fig. 2). E.coli ATCC 25922 was used as a negative control.

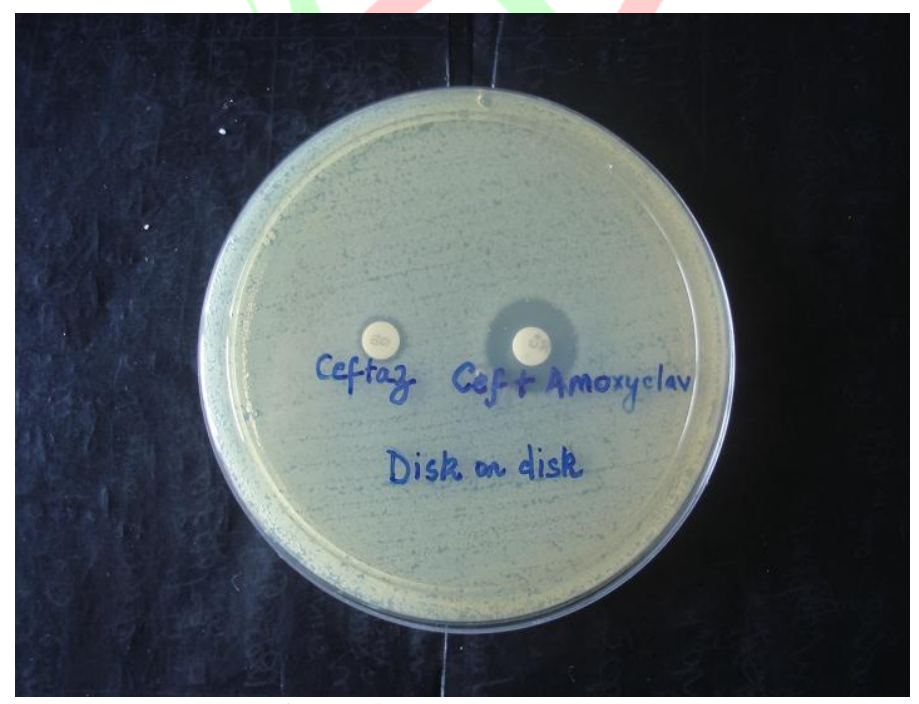

Figure 2 - Disk on disk test.

\section{RESULTS}

Maximum samples were of urine and from these ESBL production was detected in 43.5\% by DDST and 23.4\% DOD. Pus samples showed highest rate of ESBL production both by DDST (77.7\%) and DOD (44.4\%). Another significant finding was the absence of ESBL production in 4 of the blood samples received. In total ESBLs were detected in $47 \%$ of the isolates, out of which DDSTs gave a positive result in 45 (45\%) and DOD in 23 (23\%) of cases. The sample source distribution of the 100 isolates positive with screening test for ESBL production is shown in Table 1.

Maximum drug resistance was seen with ampicillin (98.7\%) followed by cefoperazone (96.6\%), co-trimoxazole (94.4\%), ciprofloxacin $(91.7 \%)$ and aminoglycosides $(67.7 \%)$. No resistance was observed for meropenem. In case of urinary isolates better results were obtained with nitrofurantoin with resistance in $44.7 \%$ cases. Resistance pattern of E.coli to individual drugs is shown in Table 2. Multiple resistance to six, five, four and three drugs was 56\%, 30\%, $9 \%$ and $5 \%$ respectively. 
IOSR Journal of Pharmacy

Vol. 2, Issue 3, May-June, 2012, PP.504-508

Table 1: Sample source distribution and ESBL production by DDST \&DOD

\begin{tabular}{|l|l|l|l|l|}
\hline $\begin{array}{l}\text { S } \\
\text { No }\end{array}$ & SOURCE & No. & $\begin{array}{l}\text { ESBL } \\
\text { BY } \\
\text { DDST } \\
(\%)\end{array}$ & $\begin{array}{l}\text { ESBL } \\
\text { BY } \\
\text { DOD } \\
(\%)\end{array}$ \\
\hline $\mathbf{1 )}$ & URINE & 78 & $\begin{array}{l}34(43 . \\
5)\end{array}$ & $\begin{array}{l}19(24 . \\
3)\end{array}$ \\
\hline $\mathbf{2 )}$ & PUS & 9 & $7(77.7)$ & $4(44.4)$ \\
\hline $\mathbf{3 )}$ & $\begin{array}{l}\text { ENDOCERVIC } \\
\text { AL } \\
\text { SWAB }\end{array}$ & 6 & $2(33.3)$ & 0 \\
\hline $\mathbf{4 )}$ & BLOOD & 4 & 0 & 0 \\
\hline $\mathbf{5 )}$ & STOOL & 3 & $2(66.6)$ & 0 \\
\hline & TOTAL & 100 & $45(45)$ & $23(23)$ \\
\hline
\end{tabular}

Table 2: Resistance pattern of ESBL producing E.coli to individual drugs.

\begin{tabular}{|l|l|}
\hline ANTIBIOTIC & RESISTANCE \\
\hline AMPICILLIN & $98.7 \%$ \\
\hline CEFOPERAZONE & $96.6 \%$ \\
\hline COTRIMOXAZOLE & $94.4 \%$ \\
\hline CIPROFLOXACIN & $91.7 \%$ \\
\hline AMINOGLYCOSIDES & $67.7 \%$ \\
\hline NITROFURANTOIN & $44.7 \%$ \\
\hline MEROPENEM & $0 \%$ \\
\hline
\end{tabular}




\section{DISCUSSION}

Many ESBL producers do not appear to be resistant by routine susceptibility testing although ESBL producers are resistant to cephalosporins and penicillins in general. It is a well known fact that diffusion testing on its own is not capable of detecting low level resistance and it should be backed up by methods specifically aimed at detecting ESBL activity. ESBL producers can be screened for by using CLSI screening criteria as this method requires no extra resources. Ceftazidime (30 $\mu$ g) is considered the best antibiotic for suspecting resistance as this antibiotic may be the only one for which the decreased resistance actually reaches the breakpoint for interpretation of resistance ${ }^{[10]}$.If any of the zone diameters in screening tests indicates suspicion for ESBL production then phenotypic confirmatory test should certainly be used which helps to ascertain the diagnosis and gives valuable information to the clinician ${ }^{[9]}$.

The results of this study showed a high frequency of ESBL producing E.coli and is in accordance with other studies in India ${ }^{[11,12]}$. DDST is easier to incorporate in any existing set up as there is no need to measure the zone sizes and it can be easily read by the presence of synergy effect. We got better results with DDST by which $45 \%$ of ESBL producers could be detected.

The DOD test on the other hand was found to be technically more difficult. This test requires petri plates to be incubated upside down relative to convention so that upper disc does not fall off and one has to measure the zone sizes of cephalosporin disc with or without clavulanic acid which will slightly prolong the time taken to read results. The results obtained with this method were not as satisfactory as compared to DDST which could be due to the displacement of the overlaid disc of co amoxiclav or due to improper diffusion of clavulanic acid from this co-amoxiclav disc as does not come in direct contact with the surface of MHA.

It is an established fact that ESBL producers show cross resistance to other antibiotics thus limiting the therapeutic option s to treat infections, as noticed in our study. Senstivity to meropenem was absolute and can be used as a treatment option in such cases. Nitrofurantoin and aminoglycosides constitute a reasonable option for treatment of urinary tract infections. Cefoperazone, cotrimoxazole and ciprofloxacin were not found to be suitable for empirical selection. Ampicillin showed even worse results and is thus not recommended as a therapeutic option.

\section{CONCLUSION}

We conclude based on our study that there is a high frequency of ESBL producers amongst E.coli in our hospital. An appropriate antibiotic policy should be framed and all clinicians be provided with local updates of microbial resistance patterns. It will help the clinicians to avoid indiscriminate and extensive use of antimicrobials especially cephalosporins. In addition, all infections with ESBL producing bacteria should be reported to hospital infection control committee so that necessary measures can be initiated to prevent the spread of MDR organisms. This will provide therapeutic benefits to the patients but also reduce the health care costs. Also, it should be mandatory for all laboratories to incorporate DDST for detection of ESBL especially for gram negative organisms as it is an convenient test to performs. 


\section{REFERENCES}

[1]. $\quad$ PA Bradford, Extended spectrum $\beta$ lactamases in the $21^{\text {st }}$ century: characterization, epidemiology and detection of this important resistance threat, Clin Microbiol Rev 2001; 14: 933-951.

[2]. M Gniadkowski, I Schneider, R Jungwirth, W Hryniewicz, A Bauernfeind, Ceftazidime-resistant Enterobacteriaceae isolates from three Polish hospitals: Identification of three novel TEM and SHV-5 type extended spectrum $\beta$ lactamases, Antimicrob Agents Chemother 1998; 42: 514-520.

[3]. $\quad$ DL Paterson, RA Bonomo, Extended spectrum $\beta$ lactamases. A clinical update, Clin Microbiol Rev 2005; 18(4):657-686.

[4]. $\quad$ AA Cagatay ,T Kocagoz, Eraksoy H. Dio-Sensimedia: A novel culture medium for rapid detection of extended spectrum $\beta$ lactamases, BMC Infect Dis 2003;22.

[5]. PB Crichton, Enterobacteriaceae: Escherichia, Klebsiella, Proteus and other genera in JGCollee, AGFraser, BP Marmion, A Simions (Eds), Mackie and Mc Cartney Practical Medical Microbiology, 14 (Churchill Livingstone, New York 1996) 361-364.

[6]. National committee for clinical Laboratory standards (NCCLS).Performance Standards for Antimicrobial Susceptibility Testing: 12 ${ }^{\text {th }}$ informational supplement, M100-512.NCCLS: Wayne 2002.

[7]. Antimicrobial Susceptibility Testing in EW Koneman, SD Allen, WM Janda, PC Schreckenberger, Winn WC ( Eds ), Color atlas and textbook of Diagnostic Microbiology, 5 (Lippincott, Philadelphia 1997):831.

[8]. RS Miles, SGB Amyes, Laboratory control of antimicrobial therapy in JG Collee, AG Fraser, BP Marmion, A Simions (Eds) ie and Mc Cartney Practical Medical Microbiology, 14(Churchill Livingstone, New York 1996)152-178.

[9]. FM Mackenzie, CA Miller, IM Gould. Comparison of screening methods of TEM and SHV-derived extended spectrum beta lactamase detection, Clin Microbiol Infect 2002; 8(11):715-724.

[10]. GP Katsanis, J Spargo, MJ Ferraro, L Sutton, GA Jacoby. Detection of Klebsiella pneumonia and Escherichia coli strains producing extended spectrum beta lactamases, J Clin Microbiol 1994; 32:691-696.

[11]. A Varaiya, J Dogra, M Kulkarni. Extended spectrum beta lactamase producing Escherichia coli and Klebsiella pneumonia in diabetic foot infection, Indian J Med Microbiol 2008; 26:281-282.

[12]. R Gadepalli, B Dhawan, V Sreenivas, A Kapil, AC Ammini, R Chaudhary . A clinic-microbiological study of diabetic foot ulcers in and Indian tertiary care hospital, Diabetes Care 2006; 29:1727-1732. 